\title{
ESTRUTURA ETÁRIA DE POPULAÇÕES DE Mauritia flexuosa L. F. (ARECACEAE) DE VEREDAS DA REGIÃO CENTRAL DE GOIÁS, BRASIL ${ }^{1}$
}

\author{
Isa Lucia de Morais Resende², Flavio Pereira dos Santos ${ }^{3}$, Lázaro José Chaves ${ }^{3}$ e Jorge Luiz do \\ Nascimento ${ }^{3}$
}

\begin{abstract}
RESUMO - A Mauritia flexuosa (buriti) é considerada espécie-chave no ambiente de veredas, sendo escassas as informações sobre sua estrutura populacional para subsidiar a elaboração de estratégias de manejo e conservação. Neste trabalho são avaliadas seis populações da espécie em áreas de veredas com diferentes graus de antropização nos municípios de Bela Vista de Goiás, Silvânia e São Miguel do Passa Quatro, GO, na região nuclear do Cerrado brasileiro. Foram coletados dados de altura de 584 indivíduos, desde plântulas até adultos, sendo amostrados 100 indivíduos por população, com exceção de uma, para a qual foram amostrados 84 indivíduos. A maioria das populações estudadas apresentou curvas de distribuição dos indivíduos em classes de altura em forma de J invertido, característica de populações autorregenerativas. A maior proporção de indivíduos mortos ocorreu no menor intervalo de classe de altura, com a maior expectativa de vida no intervalo de classe de altura entre $3,5 \mathrm{~m}$ e $7 \mathrm{~m}$.
\end{abstract}

Palavras-chave: Mauritia flexuosa, Vereda e Estrutura de populações.

\section{AGE STRUCTURE OF Mauritia flexuosa L. F. (ARECACEAE) SWAMP POPULATIONS IN THE CENTRAL REGION OF GOIÁS, BRAZIL}

\begin{abstract}
Mauritia flexuosa (buriti palm) is considered a key species in the swampy environment and little is known about their population structure to support its management and conservation strategies. This work investigated six populations of this species, from swampy areas with different degrees of human disturbance in the municipality of Bela Vista de Goiás, Silvânia and São Miguel do Passa Quatro, GO, in the nuclear region of the Brazilian Cerrado, a savannah-like vegetation. Plant height data were collected from 584 individuals, from seedlings to adults, with 100 sampled individuals per population, excluding one, in which 84 individuals were sampled. Most of the studied populations showed distribution of individuals in plant height classes according to expected inverted-J shaped curve, a self-regenerating population characteristic. The highest proportion of dead individuals occurred in the first height class and the highest life expectancy occurred in the height class with interval from $3.5 \mathrm{~m}$ to $7.0 \mathrm{~m}$.
\end{abstract}

Keywords: Mauritia flexuosa, Swamp and Population structure

\footnotetext{
${ }^{1}$ Recebido em 07.07.2010 e aceito para publicação em 15.11.2011.

${ }^{2}$ Universidade Estadual de Goiás, UEG,Unidade de Quirinópolis, Brasil. E-mail: <isamorais1@gmail.com>.

${ }^{3}$ Universidade Federal de Goiás, UFG, Agronomia, Brasil. E-mail: <lazaro.chaves@pq.cnpq.br>.
} 


\section{INTRODUÇÃO}

Na região do Cerrado, embora predominem as fitofisionomias de solos bem drenados, ocorrem também áreas úmidas, entre elas as veredas. Estas são comunidades hidrófilas formadas por dois estratos: um herbáceo-graminoso contínuo, que ocupava a maior parte da área; e outro arbóreo-arbustivo, com predominância de indivíduos da palmeira Mauritia flexuosa L. f., popularmente conhecida como buriti, com dossel entre 5\% e 10\% (RIBEIRO; WALTER, 2008).

M. flexuosa (Arecaceae) é uma palmeira definidora dos ambientes de veredas, tanto pela importância ecológica quanto pela alta densidade em comparação com as poucas espécies arbóreas que ali ocorrem. Ela serve como fonte de alimento e local de abrigo e de reprodução para diversos elementos da fauna, podendo ser considerada como uma espécie-chave nas veredas. Várias espécies de aves utilizam-na para nidificação, e os frutos são bastante apreciados por diversas espécies de psitacídeos, entre elas a Ara ararauna (KUNIY et al., 2001). Quando esses frutos caem no solo são comidos por mamíferos, incluindo desde pequenos roedores, queixadas, catitus até o maior mamífero terrestre, a anta (Tapirus terrestris) (VILLALOBOS, 1994). As flores contêm néctar, que é utilizado por himenópteros como as abelhas Trigona sp. e Apis mellifera L., as vespas Polistes sp. e Polybia sp. e as formigas Camponotus sp. e por dípteros como a mosca Ornidia obesa. Os coleópteros das famílias Nitidulidae, Mycetophagidae e Curculionidae usam as flores para reprodução, e suas larvas são grandes devoradoras de grãos de pólen (ABREU, 2001).

Alguns autores têm salientado a importância de palmeiras, entre elas $M$. flexuosa, como ecótopos naturais de triatomíneos, principalmente espécies do gênero Rhodnius (GURGEL-GONÇALVES, 2004). A espécie é fitotelmata, ou seja, o pecíolo pode armazenar grande volume de água, suportando uma abundante e relativamente rica fauna de macroinvertebrados aquáticos (NEISS, 2007).

Além da importância ecológica, $M$. flexuosa possui grande potencial de uso como fonte alternativa de renda para comunidades rurais, sendo já explorada de forma extrativista em algumas regiões. A polpa dos frutos pode ser empregada nas indústrias alimentícias, farmacêuticas e de cosméticos (ALMEIDA et al., 2008). As sementes são utilizadas no artesanato e produção de álcool combustível, o óleo é usado para fritar peixe, fabricar sabão e cosméticos e como combustível para lamparina, o pecíolo fornece material leve e macio utilizado em artesanato para confecção de brinquedos e rolha de garrafa, além de outras utilidades. Já as folhas novas ainda fechadas, conhecidas como “olhos”, são transformadas em corda, cestas, cintos, bolsas, esteiras, chapéus, sandálias, capas de agendas eredes (CYMERYS et al., 2005). Também podem ser empregadas na indústria de celulose e papel (PEREIRA et al., 2003) e na construção civil (LIMA, 2005).

Diante da importância ecológica e como alternativa econômica, nos últimos anos tem crescido o número de pesquisas envolvendo $M$. flexuosa. Entretanto, existem poucos estudos sobre a estrutura de populações dessa palmeira(CARDOSO et al., 2002; SOUZA, 2005; JARDIM et al., 2007; SAMPAIO et al., 2008; MACHADO; SILVEIRA, 2009). O estudo da estrutura etária de populações permite avaliar a capacidade autorregenerativa, abundância, distribuição de tamanho, distribuição espacial e grupos ecológicos, entre outros processos que ocorrem em nível populacional. Essas informações podem subsidiar ações de conservação e programas de recuperação de áreas degradadas (AQUINO et al., 2007).

Nesse contexto, o objetivo deste estudo foi avaliar a estrutura etária de populações de $M$. flexuosa em veredas da região central de Goiás, visando subsidiar programas de manejo e de conservação.

\section{MATERIAL E MÉTODOS}

Depois da realização de estudos prévios com o uso de imagens de satélite e viagens de reconhecimento, foram escolhidas seis veredas situadas em ambientes conservados e antropizados. Foram consideradas conservadas as veredas que apresentavam vegetação nativa no entorno superior a $50 \mathrm{~m}$ de largura, conforme determina a Lei no . 7.803, de 18 de julho de 1983 (BRASIL, 1992). As veredas com vegetação nativa ausente ou inferior a $50 \mathrm{~m}$ no entorno foram denominadas antropizadas.

O estudo foi realizado em seis veredas (Tabela 1), as quais fazem parte da vegetação ripária de afluentes do rio do Peixe, o qual é afluente do rio Corumbá, da bacia do rio Paranaíba (Figura 1), e estão localizadas em propriedades particulares.

A vereda conservada de Bela Vista de Goiás faz transição com o Cerradão e apresenta represas abaixo da área de estudo para dessedentação do gado, sendo 
Tabela 1 - Localização e tipo de vegetação no entorno das veredas conservadas (C), circundadas por pastagem (P) e circundadas por lavoura (L), em Bela Vista de Goiás (C1, P1 e L1), Silvânia (C2 e P2) e São Miguel do Passa Quatro (L2), GO.

Table 1 - Location and type of vegetation around the conserved swamps $(C)$, surrounded by pasture $(P)$ and surrounded by farming (L) in Bela Vista de Goiás (C1, P1, L1), Silvânia (C2 and P2) and São Miguel do Passa Quatro (L2), GO.

\begin{tabular}{|c|c|c|c|c|}
\hline Vereda & Município & Latitude S & Longitude W & Entorno \\
\hline C1 & Bela Vista de Goiás & $17^{\circ} 00^{\prime} 63^{\prime \prime}$ & $48^{\circ} 47^{\prime} 33^{\prime \prime}$ & Vegetação nativa \\
\hline $\mathrm{P} 1$ & Bela Vista de Goiás & $17^{\circ} 01^{\prime} 27^{\prime \prime}$ & $48^{\circ} 48^{\prime} 05^{\prime \prime}$ & Pastagem \\
\hline $\mathrm{L} 1$ & Bela Vista de Goiás & $17^{\circ} 00^{\prime} 29^{\prime \prime}$ & $48^{\circ} 47^{\prime} 13^{\prime \prime}$ & Lavoura anual \\
\hline $\mathrm{C} 2$ & Silvânia & $16^{\circ} 49^{\prime} 34^{\prime \prime}$ & $48^{\circ} 41^{\prime} 35^{\prime \prime}$ & Vegetação nativa \\
\hline P 2 & Silvânia & $16^{\circ} 48^{\prime} 15^{\prime \prime}$ & $48^{\circ} 44^{\prime} 39^{\prime \prime}$ & Pastagem \\
\hline $\mathrm{L} 2$ & S. Miguel do Passa Quatro & $16^{\circ} 52^{\prime} 28^{\prime \prime}$ & $48^{\circ} 43^{\prime} 27^{\prime \prime}$ & Lavoura anual \\
\hline
\end{tabular}

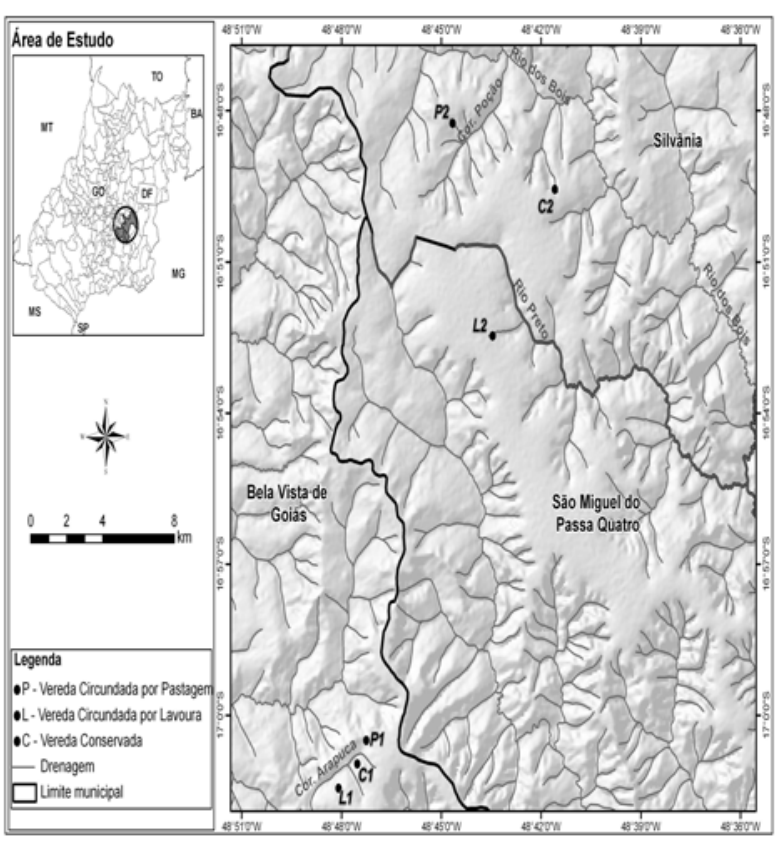

Figura 1 - Localização das seis áreas de estudo em Bela Vista de Goiás, Silvânia e São Miguel do Passa Quatro, GO.

Figure 1 - Location of six analized areas in Bela Vista de Goiás, Silvânia and São Miguel do Passa Quatro, GO.

observados sinais de perturbação como marcas de fogo no estipe dos buritis e, raras vezes, pisoteio da vegetação pelo gado. A vereda conservada de Silvânia fazia transição com o Cerrado sentido restrito e se encontrava cercada, não sendo observados vestígios de animais domésticos no local.

Entre as veredas antropizadas, considerando o período de amostragem, duas apresentavam seu entorno ocupado por pastagens cultivadas, com predominância de Brachiaria sp., enquanto as outras duas tinham o entorno submetido à atividade agrícola de cultivo de grãos (especialmente as culturas de milho e soja). Entre as veredas estudadas, as únicas que não apresentavam cerca em uma das margens ou em seu entorno foram a circundada por pastagem em Silvânia e a circundada por lavoura em Bela Vista de Goiás. Na vereda circundada por pastagem em Silvânia foram observadas plantas jovens de buritis com sinais de que as folhas tinham sido comidas pelo gado.

O clima da região, segundo a classificação de Köppen, é do tipo Aw, megatérmico, com chuvas de verão e estação relativamente seca no inverno. Os valores da temperatura média variam entre 19 e $28^{\circ} \mathrm{C}$, e a pluviosidade média é inferior a $2.000 \mathrm{~mm} / \mathrm{ano}$ (INMET, 2009). Os locais de amostragem apresentam relevo plano a suave ondulado, com altitudes variando de 910 a $1.040 \mathrm{~m}$.

Normalmente, em espécies arbóreas dicotiledôneas estudos de estrutura etária de populações são realizados utilizando-se medidas de altura e de diâmetro, uma vez que o diâmetro aumenta com a idade das árvores. Entretanto, neste estudo foram utilizados somente dados de altura pelo fato de a espécie ser uma palmeira que define o diâmetro muito jovem, não apresentando variação considerável com o passar do tempo.

As coletas foram realizadas em três dias, sendo realizadas em maio de 2008, fevereiro de 2009 e janeiro de 2010. Foram coletados dados de altura de 584 indivíduos de $M$. flexuosa, desde plântulas até adultos, sendo em cada vereda amostrados 100 indivíduos, com exceção da vereda circundada por lavoura em São Miguel de Passa Quatro, onde só foi possível amostrar 84 indivíduos, porque a vereda abrigava uma população com menor número de indivíduos. Estes foram amostrados

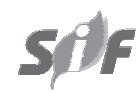

Revista Árvore, Viçosa-MG, v.36, n.1, p.103-112, 2012 
aleatoriamente, medindo-se todas as plantas de determinada faixa da vereda até atingir o número estabelecido. A altura das plantas adultas foi obtida por projeção de triângulo retângulo, medindo-se as distâncias da árvore até uma haste de referência e desta até o observador, com o auxílio de uma trena de 50 m. Os indivíduos jovens foram medidos diretamente com o uso de uma trena.

Os intervalos de classes para a confecção dos histogramas de altura foram obtidos utilizando-se a fórmula: A/K, em que A representa a amplitude dos valores de altura e $\mathrm{K}$ indica uma constante definida pelo algoritmo de Sturges, que consiste em $1+3,3$ $\times \log _{10} \mathrm{n}$, em que $\mathrm{n}$ é o número total de indivíduos amostrados (BONINI; BONINI, 1972). O resultado dessa razão (A/K) representa o valor do incremento empregado para definir os intervalos de classes.

Os dados foram analisados utilizando-se a distribuição em classes de altura e o cálculo do Quociente de De Liocourt (MEYER, 1952). O Quociente “q” de De Liocourt, no limite entre duas classes, é obtido pela divisão do número de indivíduos da classe de altura pelo número de indivíduos da classe anterior. Algebricamente, pode ser estimado pela linearização da função $Y_{i}=k e-^{a X i}$, ou seja, In $Y_{i}=k-a X_{i}$, sendo $Y_{i}$ a frequência de indivíduos na classe i e $\mathrm{X}_{\mathrm{i}}$ o valor central da classe. O Quociente de De Liocourt é obtido por e- ${ }^{\text {aw }}$, sendo w o valor da amplitude de classe (MURPHY; FARRAR, 1981).

A significância dos valores do coeficiente de regressão foi verificada pelo teste F por meio de análise de regressão, comparando o quadrado médio da regressão com o quadrado médio dos desvios da regressão (STEEL; TORRIE, 1980). A comparação entre os valores dos coeficientes de determinação $\left(\mathrm{R}^{2}\right)$ de cada população foi feita usando-se a transformação $R$ para $Z$ e verificando a significância diretamente na distribuição normal padronizada.

Para estimar as taxas de mortalidade e a esperança de vida, foram utilizadas tabelas de vida baseadas nas classes de altura das populações de $M$. flexuosa. Para a elaboração das tabelas de vida, determinaram-se os valores de número de sobreviventes $\left(\mathrm{l}_{\mathrm{x}}\right)$, número de indivíduos mortos $\left(\mathrm{d}_{\mathrm{x}}\right.$ ) durante o intervalo etário, estrutura etária $\left(E_{x}\right)$, número de indivíduos vivos entre um intervalo de classe de altura e outro e esperança de vida $\left(\mathrm{e}_{\mathrm{x}}\right)$, em que $\mathrm{x}$ é a classe etária,

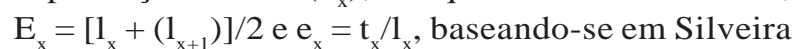
Neto et al. (1976).

\section{RESULTADOS}

Nas veredas estudadas, a maioria dos indivíduos adultos de $M$. flexuosa encontrava-se distribuída na região mais úmida, denominada região de fundo, formando um alinhamento em que o canal de drenagem estava se definindo.

Os indivíduos foram separados em oito classes de altura, por intervalos de classe igual a 3,5 m. Aproximadamente $40 \%$ dos indivíduos apresentaram alturas inferiores a 3,5 m, e cerca de 1,5\% já havia atingido alturas entre 17,5 m e 28,0 m. Apenas a população da vereda circundada por lavoura, em Bela Vista de Goiás (Figura 2L1), apresentou indivíduos nas duas classes maiores de altura.

Embora tenha ocorrido variação nos valores encontrados nas razões “q”, há uma tendência para o padrão “J - invertido” para a estrutura das alturas das populações (Figura 2), com exceção das populações das veredas circundadas por pastagem, de Bela Vista de Goiás (Figura 2P1), e a conservada, de Silvânia (Figura 2C2), com os valores de coeficiente de determinação $\left(R^{2}\right)$ na ordem de 0,1079 e 0,2186, respectivamente.

Todas as populações apresentaram coeficiente $\mathrm{q}_{1}$, da primeira para a segunda classe, variando em torno de 0,5 ou com valores mais baixos e grande número de indivíduos na menor classe de altura.

Houve maior proporção de indivíduos mortos de $M$. flexuosa no menor intervalo de classe de altura, com a maior expectativa de vida no intervalo de classe de altura entre 3,5 m e $7 \mathrm{~m}$ (Tabela 2).

As populações das veredas circundadas por lavoura em Bela Vista de Goiás (Figura 2L1) e por pastagem em Silvânia (Figura 2P2) apresentaram baixo número de indivíduos na menor classe de tamanho, em comparação com as outras populações. Essas populações exibiram maior número de indivíduos nas classes intermediárias de altura. Embora a primeira classe de altura tenha o maior (L1) ou o segundo maior número de indivíduos (P2), a diferença no valor é pequena 

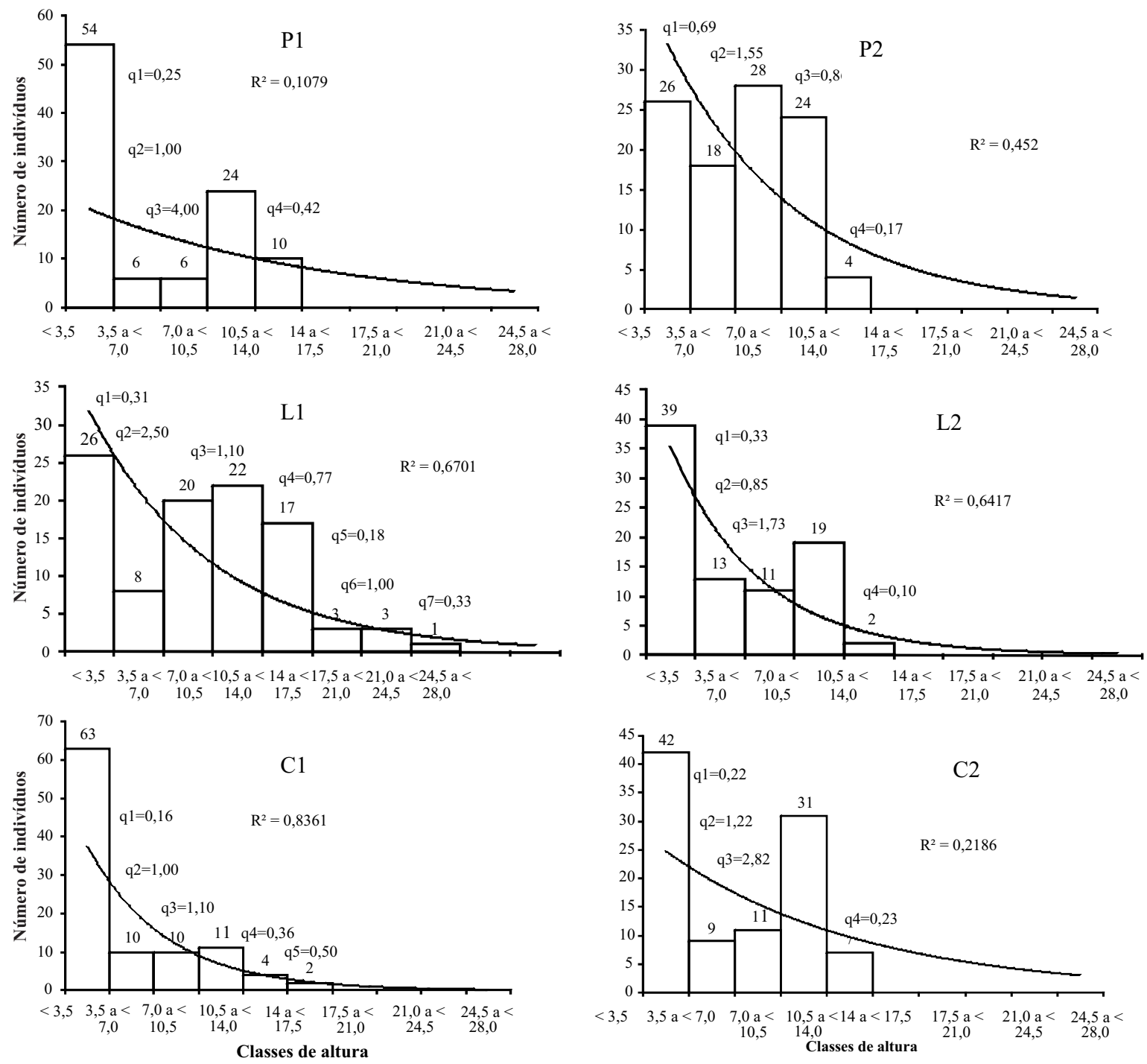

Figura 2 - Distribuição nos intervalos de classe de altura dos indivíduos de Mauritia flexuosa sob curva de ajuste “J invertido”

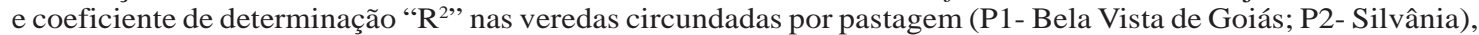
circundadas por lavoura (L1- Bela Vista de Goiás; L2- São Miguel do Passa Quatro) e conservadas (C1- Bela Vista de Goiás; C2- Silvânia).

Figure 2 - Distribution into height class ranges of Mauritia flexuosa individuals under curve fit in "inverted J" shape and "R2" coefficient of determination in the swamps surrounded by pasture (P1-Bela Vista de Goiás; Silvânia-P2), surrounded by farming (L1 - Bela Vista de Goiás; L2-São Miguel do Passa Quatro) and the conserved ones (C1-Bela Vista de Goiás; C2-Silvânia).

para o número de indivíduos encontrados nas classes intermediárias. Essas populações são mais maduras, com a maioria dos indivíduos em estádio avançado de reprodução, diferindo da situação encontrada nas outras veredas, as quais apresentavam populações predominantemente jovens.
Observou-se que as razões $\mathrm{q}_{2}$ e $\mathrm{q}_{3}$ foram altas em todas as populações.

Estatisticamente, houve diferença significativa apenas entre as populações da vereda conservada e a circundada por pastagem $(p=0,0276)$, ambas de

Revista Árvore, Viçosa-MG, v.36, n.1, p.103-112, 2012 
Tabela 2 - Tabela de vida baseada no intervalo de classes de altura dos indivíduos de Mauritia flexuosa nas veredas conservadas (C), circundadas por pastagem (P) e circundadas por lavoura (L), em Bela Vista de Goiás (C1, P1 e L1), Silvânia (C2 e P2) e São Miguel do Passa Quatro (L2), GO. $l x=n^{\circ}$ de sobreviventes, $d x=n^{\circ}$ de mortos, $q x=$ taxa de mortalidade e ex = expectativa de vida.

Table 2 - Life table based on the range of individuals height classes of Mauritia flexuosa in the conserved swamps (C), surrounded by pasture $(P)$ and surrounded by farming $(L)$ in Bela Vista de Goiás (C1, P1, L1), Silvânia (C2 and P2) and São Miguel do Passa Quatro (L2), GO. lx = number of survivors; $d x=$ No dead; $q x=$ mortality rate and ex $=$ life expectancy.

\begin{tabular}{|c|c|c|c|c|c|c|c|c|c|c|c|c|c|c|c|}
\hline \multirow[t]{2}{*}{ Classes } & \multicolumn{3}{|c|}{$\mathrm{n}^{\circ}$ de indivíduos } & \multicolumn{3}{|c|}{$\mathrm{lx}$} & \multicolumn{3}{|c|}{$\mathrm{dx}$} & \multicolumn{3}{|c|}{$\mathrm{qx}$} & \multicolumn{3}{|c|}{$\mathrm{ex}$} \\
\hline & $\overline{\mathrm{C} 1}$ & $\mathrm{P} 1$ & $\overline{\mathrm{L} 1}$ & C1 & P1 & L1 & $\mathrm{C} 1$ & $\mathrm{P} 1$ & $\mathrm{~L} 1$ & C1 & P1 & L1 & C1 & $\mathrm{P} 1$ & L1 \\
\hline$<3,5$ & 63 & 54 & 26 & 1000 & 1000 & 1000 & 841 & 889 & 692 & 0,84 & 0,89 & 0,69 & 1,09 & 1,35 & 3,35 \\
\hline $3,5 \mathrm{a}<7,0$ & 10 & 6 & 8 & 159 & 111 & 308 & 0 & 0 & -461 & 0,00 & 0,00 & $-1,50$ & 3,20 & 7,17 & 8,74 \\
\hline 7,0 a $<10,5$ & 10 & 6 & 20 & 159 & 111 & 769 & -16 & -333 & -77 & $-0,10$ & $-3,00$ & $-0,10$ & 2,20 & 6,17 & 2,80 \\
\hline 10,5 a $<14,0$ & 11 & 24 & 22 & 175 & 444 & 846 & 112 & 259 & 192 & 0,64 & 0,58 & 0,23 & 1,04 & 0,92 & 1,59 \\
\hline $14 \mathrm{a}<17,5$ & 4 & 10 & 17 & 63 & 185 & 654 & 31 & 185 & 539 & 0,49 & 1,00 & 0,82 & 1,01 & 0,50 & 0,91 \\
\hline 17,5 a $<21,0$ & 2 & 0 & 3 & 32 & - & 115 & 32 & - & 0 & 1,00 & - & 0,00 & 0,50 & - & 1,83 \\
\hline 21,0 a $<24,5$ & 0 & 0 & 3 & - & - & 115 & - & - & 77 & - & - & 0,67 & - & - & 0,83 \\
\hline \multirow[t]{2}{*}{24,5 a $<28,0$} & 0 & 0 & 1 & - & - & 38 & - & - & 38 & - & - & 1,00 & - & - & 0,50 \\
\hline & $\mathrm{C} 2$ & $\mathrm{P} 2$ & $\mathrm{~L} 2$ & $\mathrm{C} 2$ & $\mathrm{P} 2$ & $\mathrm{~L} 2$ & $\mathrm{C} 2$ & $\mathrm{P} 2$ & $\mathrm{~L} 2$ & $\mathrm{C} 2$ & $\mathrm{P} 2$ & $\mathrm{~L} 2$ & $\mathrm{C} 2$ & $\mathrm{P} 2$ & $\mathrm{~L} 2$ \\
\hline$<3,5$ & 42 & 26 & 39 & 1000 & 1000 & 1000 & 786 & 308 & 667 & 0,79 & 0,31 & 0,67 & 1,88 & 3,35 & 1,65 \\
\hline 3,5 a $<7,0$ & 9 & 18 & 13 & 214 & 692 & 333 & -48 & -385 & 51 & $-0,22$ & $-0,56$ & 0,15 & 5,95 & 3,61 & 2,96 \\
\hline 7,0 a $<10,5$ & 11 & 28 & 11 & 262 & 1077 & 282 & -476 & 154 & -205 & $-1,82$ & 0,14 & $-0,73$ & 3,95 & 1,50 & 2,41 \\
\hline 10,5 a $<14,0$ & 31 & 24 & 19 & 738 & 923 & 487 & 571 & 769 & 436 & 0,77 & 0,83 & 0,90 & 0,73 & 0,67 & 0,60 \\
\hline $14 \mathrm{a}<17,5$ & 7 & 4 & 2 & 167 & 154 & 51 & 167 & 154 & 51 & 1,00 & 1,00 & 1,00 & 0,50 & 0,50 & 0,50 \\
\hline
\end{tabular}

Bela Vista de Goiás, indicando que a população da vereda conservada se ajustou melhor ao padrão esperado.

\section{DISCUSSÃO}

Em estudo realizado por Cardoso et al. (2002), 95\% dos indivíduos de buritis (jovens e adultos) foram encontrados no fundo da vereda. Acredita-se que o predomínio de adultos nessa região, além da preferência por um solo hidromórfico para a germinação das sementes (CYMERYS et al., 2005), seja devido à incidência de fogo nas veredas ao longo dos tempos, eliminando o desenvolvimento de adultos nas regiões de borda e meio. Observou-se que nessas últimas regiões havia apenas plântulas e, ou, jovens dessa palmeira, os quais se estabeleceram depois da passagem do fogo, sendo verificados sinais de incêndio em alguns buritizeiros. Mesmo sendo as veredas ambientes úmidos, em anos com estação seca mais intensa o fogo pode se alastrar devido ao predomínio de espécies de Poaceae e Cyperaceae, que compõem a maior parte do estrato herbáceo.

A carência ou ausência de indivíduos nas classes maiores de tamanho pode indicar grande perturbação antrópica no passado (CARVALHO et al., 2007). Embora a distribuição de altura não corresponda ao padrão esperado para populações equilibradas, essas duas populações apresentam boa possibilidade de recuperação devido à grande quantidade de indivíduos na primeira classe de altura e pelo bom estado de conservação das veredas. Uma distribuição de altura de indivíduos de M. flexuosa, fora do padrão “J invertido", foi obtida no levantamento realizado em uma floresta de várzea, na Ilha do Combu, em Belém, Pará, por Jardim et al. (2007). Nessa floresta de várzea, a maior parte das alturas encontradas foi intermediária: 43,33\% $(n=13)$ dos indivíduos distribuídos no intervalo de classe de 5,1 m a $10,0 \mathrm{~m}$ e $36,67 \%(\mathrm{n}=11)$ no intervalo de $10,1 \mathrm{~m}$ a 20,0 m, num total de 30 indivíduos amostrados. Já nos estudos de Sampaio et al. (2008) e Machado e Silveira (2009) as populações dessa espécie apresentaram estrutura em formato de $\mathrm{J}$ invertido.

Houve bastante discrepância na relação entre as primeiras classes de altura e as subsequentes, o que indica que poderá haver acréscimo de indivíduos nas populações estudadas. A maior concentração de indivíduos nas primeiras classes de altura pode caracterizar um banco de plântulas-estoque, o que é um padrão em florestas tropicais estáveis com idade e composição de espécies variadas (SCOLFORO et al., 1998). Cada classe de altura representa uma etapa

Revista Árvore, Viçosa-MG, v.36, n.1, p.103-112, 2012 
da regeneração da fração do povoamento de uma mesma espécie ou de uma comunidade com a altura superior à dessa classe (ROLLET, 1978). À medida que aumenta o tamanho da classe, a frequência diminui até atingir o menor valor na maior classe de diâmetro, ou seja, a frequência decresce segundo uma progressão geométrica constante (MEYER et al., 1961), caracterizando uma curva do tipo exponencial ou denominada ' J'" invertido (SCOLFORO, 1998).

Para que uma população esteja em equilíbrio, é necessária grande produção de sementes, germinação satisfatória e taxa de mortalidade decrescente nas idades mais avançadas (DAUBERNMIRE, 1968). A elevada produção de frutos por $M$. flexuosa garante a manutenção de um banco de plântulas e o consequente recrutamento delas em estágio adulto, sendo o grande número de indivíduos jovens uma estratégia adaptativa importante para a continuidade temporal da espécie no local.

Segundo Paula-Fernandes (2001), uma palmeira feminina de buriti produz de um a nove cachos, e cada cacho contém de 600 a 1.200 frutos. De acordo com Martins (2010), a qual avaliou a produção das populações de buritis nas três veredas de Bela Vista de Goiás, as médias do número de frutos por cacho e de massa foram, respectivamente, de 595,33 e 55,44 g na vereda circundada por lavoura, 436 e 60,02 g na vereda circundada por pastagem e 397,33 e 49,79 g na vereda conservada. Embora tenha ocorrido elevada produção de frutos, verificou-se maior número de indivíduos mortos nos estádios mais jovens (CARDOSO et al., 2002), o que é corroborado pela grande queda no número de indivíduos da primeira classe de altura para a segunda nas populações estudadas (Figura 2). A maioria das populações de espécies tropicais tem a mortalidade concentrada nas classes menores (SOLBRIG, 1981), e as causas podem ser a herbivoria ou a incidência de doenças e a competição entre plântulas ou entre plântulas e indivíduos adultos (HOWE, 1990). No caso de $M$. flexuosa, além desses fatores, existe outro bastante específico, que é provocado pelo impacto mecânico da queda de folhas de indivíduos adultos, as quais apresentam grandes bainhas e podem causar a mortalidade de plântulas sob a planta-mãe (REIS et al., 1996; CARDOSO et al., 2002).

Pode-se inferir que o baixo valor de indivíduos jovens das populações das veredas circundadas por lavoura em Bela Vista de Goiás e por pastagem em Silvânia, em comparação com o encontrado nas outras populações, seja devido ao intenso pastejo e pisoteio de animais domésticos. As duas veredas não se encontravam cercadas, observando-se a entrada constante de animais domésticos. A vereda de Bela Vista de Goiás, embora circundada por lavoura, sofria interferência do gado, que era colocado na época da palhada. Também, foram observados sinais de porcos, que eram soltos regularmente no local para se alimentarem. Além disso, havia a irregularidade no relevo ocasionada pela construção de pequenas represas, que acumulavam água, principalmente na época chuvosa, permitindo o estabelecimento apenas de plantas aquáticas. O buriti cresce em condições edáficas e de umidade especiais, apresentando raízes do tipo pneumatóforo, que suprem a falta de oxigênio nos brejos, mas elas podem ser sufocadas por assoreamento (POTT; POTT, 2004) ou inundação, como a ocasionada pela construção de barragens, o que causa a morte da planta. O reduzido número de indivíduos na faixa etária mais jovem das populações das veredas circundadas por lavoura em Bela Vista de Goiás e por pastagem em Silvânia pode indicar impacto recente e o comprometimento da sobrevivência dessas populações.

As causas das diferenças na composição etária das populações podem estar relacionadas com a variação do uso e ocupação do solo no entorno dos ecossistemas estudados. Nas veredas, o solo e a água interagem de forma dinâmica, influenciando reciprocamente em suas propriedades e na ocorrência e distribuição da flora (OLIVEIRA et al., 2009). Para Ramos et al. (2006), as diferenças observadas em certas propriedades do solo nos ambientes de vereda são condicionadas por fatores como: origem e intensidade de deposição dos sedimentos nas veredas, influência do nível de estabilidade do lençol freático e a própria ação antrópica. As condições de drenagem, bem como as variações dos teores e os estoques de nutrientes do solo, podem ser impactadas pelas práticas adotadas na utilização das terras adjacentes, com potencial alteração da estrutura e funções desses ecossistemas (LIMA; GUILHERME, 2002; SOUSA, 2009).

O fato de as razões q2 e q3 terem sido altas indica que as populações amostradas, em um passado próximo, sofreram perturbação que interferiu no recrutamento de indivíduos para essas classes. Essa perturbação pode ter sido na polinização e, ou, fecundação, produção de frutos, germinabilidade das sementes e, ou, estabelecimento das plântulas.

Revista Árvore, Viçosa-MG, v.36, n.1, p.103-112, 2012 
A diferença significativa apenas entre as populações da vereda conservada e a circundada por pastagem, ambas em Bela Vista de Goiás, indica que a população da vereda conservada se ajustou melhor ao padrão esperado.

A maioria das populações estudadas apresentou curvas de distribuição dos indivíduos em classes de altura em forma de J invertido, característica de populações autorregenerativas. A maior proporção de indivíduos mortos ocorreu no menor intervalo de classe de altura, com a maior expectativa de vida no intervalo de classe de altura entre $3,5 \mathrm{~m}$ e $7 \mathrm{~m}$.

Devido à importância ecológica da $M$. flexuosa, sugere-se que estudos acerca da estrutura de populações dessa palmeira sejam incentivados, visando ampliar o conhecimento sobre seu comportamento ecológico e subsidiar o manejo e conservação do ambiente de veredas.

\section{AGRADECIMENTOS}

À CAPES, pela concessão da bolsa de doutorado à primeira autora; ao CNPq, pela concessão da bolsa de iniciação científica ao segundo autor e de produtividade em pesquisa ao terceiro; aos revisores anônimos, pela leitura crítica do manuscrito; e ao CNPq/CTHidro, Edital 044/2006, pelo apoio financeiro.

\section{REFERÊNCIAS}

ABREU, S. A. B. Biologia reprodutiva de Mauritia flexuosa L. (Arecaceae) em vereda no município de UberlândiaMG. 2001. 87f. Dissertação (Mestrado em Ecologia e Conservação dos Recursos Naturais) - Universidade Federal de Uberlândia, Uberlândia, 2001.

ALMEIDA, S. P. ; COSTA, T. S. A.; SILVA, J. A. Frutas nativas do Cerrado caracterização físicoquímica e fonte potencial de nutrientes. In: SANO, S. M.; ALMEIDA, S. P.; RIBEIRO, J. F. (Eds.). Cerrado: ecologia e flora. Brasília: Embrapa Cerrados/Embrapa Informação Tecnológica, 2008. p.351-381.

AQUINO, F. G.; WALTER, B. M. T.; RIBEIRO, J. F. Dinâmica de populações de espécies lenhosas de Cerrado, Balsas, Maranhão. Revista Árvore, v.31, n.5, p.793-803, 2007.

Revista Árvore, Viçosa-MG, v.36, n.1, p.103-112, 2012
BRASIL. Resoluções CONAMA de 1984 a 1991. 4.ed. Brasília: SEMAM / IBAMA, 1992. 245p.

BONINI, E. E.; BONINI, S. E. Estatística teórica e exercícios. São Paulo: Loyola, 1972.

CARDOSO, G. L.; ARAÚJO, G. M.; SILVA, S. A. Estrutura e dinâmica de uma população de Mauritia flexuosa L. (Arecaceae) em vereda na Estação Ecológica do Panga, Uberlândia, MG. B. Boletim do Herbário Ezechias Paulo Heringer, v.9, p.34-48, 2002.

CARVALHO, F. A.; NASCIMENTO, M. T.; BRAGA, J. M. A. Estrutura e composição florística do estrato arbóreo de um remanescente de Mata Atlântica Submontana no Município de Rio Bonito, RJ, Brasil (Mata Rio Vermelho). Revista Árvore, v.31, n.4, p.717-730, 2007.

CYMERYS, M.; PAULA-FERNANDES, N. M. ; RIGAMONTE-AZEVEDO, O. C. Buriti Mauritia flexuosa L.f. In: SHANLEY, P.; MEDINA, G. (Eds.). Frutíferas e plantas úteis na vida Amazônica. Belém: CIFOR, Imazon, 2005. p.181-187.

DAUBERNMIRE, R. Plant communities: a textbook of plant synecology. New York: Haper e Row Publisher, 1968.

GURGEL-GONÇALVES, R. et al. Distribuição espacial de populações de triatomíneos (Hemiptera: Reduviidae) em palmeiras da espécie Mauritia flexuosa no Distrito Federal, Brasil. Revista da Sociedade Brasileira de Medicina Tropical, v.37, n.3, p.241-247, 2004.

HOWE, H. F. Survival and growth of juvenile Virola surinamensis in Panama: effects of herbivory and canopy closure. Journal of Tropical Ecology, v.6, p.259-280, 1990.

INSTITUTO NACIONAL DE METEOROLOGIA INMET. Gráficos e normais climatológicas. Brasília: 2009. Disponível em: <http://www.inmet.gov.br/html/clima.php>. Acesso em: 22 jan. 2010.

JARDIM, M. A. G. et al. Diversidade e Estrutura de Palmeiras em Floresta de Várzea do Estuário Amazônico. Amazônia: Ciência e Desenvolvimento, v.2, n.4, p.67-84, 2007. 
KUNIY, A. A.; YAMASHITA, C.; GOMES, E. P. C. Estudo do aproveitamento de frutos da palmeira jerivá (Syagrus romanzoffiana) por Anadorhynchus hyacinthinus, A. leari e Ara ararauna. Ararajuba, v.9, n.2, p.19-123, 2001.

LIMA, J. M.; GUILHERME, L. R. G. Recursos naturais renováveis e impacto ambiental: solo. Lavras: UFLA/FAEPE, 2002. 40p.

LIMA, J. P. Modelagem e teste de condutividade térmica em placa de gesso e fibra vegetal, Mauritia vinifera Martius, para uso na construção civil. 2005. Dissertação (Mestrado em Engenharia Mecânica) - Universidade Estadual de Campinas, Campinas, 2005.

MACHADO, F. S.; SILVEIRA, M. Estrutura populacional e aspectos etnobotânicos de Mauritia flexuosa l. F. (buriti, Arecaceae) na Amazônia Sul Ocidental, Acre. In: CONGRESSO DE ECOLOGIA DO BRASIL, 9., 2009, São Lourenço. Anais... São Lourenço: 2009.

MARTINS, M. L. Fenologia, produção e pós-colheita de frutos de buriti (Mauritia flexuosa L. f.) em três veredas do Cerrado no estado de Goiás. 2010. 144f. Dissertação (Programa de Pós-Graduação em Agronomia) - Universidade Federal de Goiás, Goiânia, 2010.

MEYER, H. A. Structure, growth, and drain in balanced uneven-aged forests. Journal of Forestry, v.50, p.85-92, 1952.

MEYER, A. H. et al. Forest management. New York: Ronald, 1961. 282p.

MURPHY, P. A.; FARRAR, R. M. A test of exponential distribution for stand structure definition in uneven-aged loblolly-shortleaf pine stands.

Washington: US Department of Agriculture Forest Service, 1981. 4p. (Research Paper SO-164)

NEISS, U. G. Estrutura da Comunidade de Macroinvertebrados Aquáticos Associados a Mauritia flexuosa Linnaeus (1782) (Arecaceae), Fitotelmata, na Amazônia Central, Brasil. 2007. 89f. Dissertação (Mestrado em Ciências Biológicas) Instituto Nacional de Pesquisas da Amazônia INPA, Manaus, 2007.
OLIVEIRA, G. C.; ARAÚJO, G. M.; BARBOSA, A. A. A. Florística e zonação de espécies vegetais em veredas no Triângulo Mineiro, Brasil.

Rodriguésia, v.60, p.1077-1085, 2009.

PAULA-FERNANDES, N. M. Estratégias de Produção de Sementes e Estabelecimento de Plântulas de Mauritia flexuosa L. f. (Arecaceae) no Vale do Acre/Brasil. 2001. 207f. Tese (Doutorado em Ciências Biológicas (Botânica)) - Instituto Nacional de Pesquisas da Amazônia - INPA, Manaus. 2001.

PEREIRA, S. J. et al. Celulose de buriti (Mauritia vinifera Martius) Buriti (Mauritia vinifera Martius) pulp. Scientia Forestalis, n.63, p.202-213, 2003.

POTT, V. J.; POTT, A. Buriti - Mauritia

flexuosa. Fauna e Flora do Cerrado. Campo Grande: 2004. Disponível em: < http:// www.cnpgc.embrapa.br/ rodiney/series/buriti/ buriti.htm>. Acesso em: 19 de fev. de 2010.

RAMOS, M. V. V. et al. Veredas do Triângulo Mineiro: solos, água e uso. Ciência e Agrotecnologia, v.30, n.2, p.283-293, 2006.

REIS, A.; et al. Demografia de Euterpe edulis Martius (Arecaceae) em uma floresta ombrófila densa montana, em Blumenau (SC). Sellowia, v. 45, n. 48, p. 13-45, 1996.

RIBEIRO, J. F.; WALTER, B. M. T. Fitofisionomias do bioma Cerrado. In: SANO, S. M.; ALMEIDA, S. P.; RIBEIRO, J. F. (Eds.). Cerrado: ecologia e flora. Brasília: Embrapa Cerrados/Embrapa Informação Tecnológica, 2008. p.151-212.

ROLLET, B. Arquitetura e crescimento das florestas tropicais. Belém: SUDAN, 1978. 22p.

SAMPAIO, M. B.; SCHMIDT, I. B.; FIGUEIREDO, I. B. Harvesting Effects and Population Ecology of the Buriti Palm (Mauritia flexuosa L. f., Arecaceae) in the Jalapão Region, Central Brazil. Economic Botany, v.62, n.2, p.171-181, 2008.

SCOLFORO ,J. R. S.; PULZ, F. A.; MELO, J. M. Modulagem da produção, idade das florestas nativas, distribuição espacial das espécies e a análise estrutural. In: SCOLFORO, J. R. S. (Org.). Manejo florestal. Lavras: UFLA/FAEPE, 1998. p.189-246.

Revista Árvore, Viçosa-MG, v.36, n.1, p.103-112, 2012 
SILVEIRA NETO, S.; NAKANO BARBIN, O. D.; VILLA NOVA, N. A. Manual de ecologia dos insetos. Piracicaba: Agronômica Ceres, 1976. 419p.

SOLBRIG, O. T. Studies on the population biology of the genus Viola. II. The effect of plant size on fitness in Viola sororia. Evolution, v.35, p.1080-1093, 1981.

SOUSA, R. F. Atributos químicos e textura do solo em veredas conservadas e antropizadas no bioma Cerrado. 2009. 71f. Dissertação (Mestrado em Agronomia) - Universidade Federal de Goiás, Goiânia, 2009.
SOUZA, M. E. B. Caracterização Estrutural de Populações de Palmeiras com Ocorrência no Parque Ecológico do Município de Belém. 2005. 87f. Dissertação (Mestrado em Ciências Florestais) - Universidade Federal Rural da Amazônia, Belém, 2005.

STEEL, R. G. D.; TORRIE, J. H. Principles and procedures of statistics: a biometrical approach. 2.ed. New York: McGraw-Hill Book, 1980. 633p.

VILLALOBOS, M. P. Guilda de frugívoros associada com o buriti (Mauritia flexuosa: Palmae) numa vereda no Brasil

Central. 1994. 99f. Dissertação (Mestrado em Ecologia) - Universidade de Brasília, Brasília, 1994. 